\title{
MEAN VALUE THEOREMS FOR GENERALIZED RIEMANN DERIVATIVES
}

\author{
J. M. ASH AND R. L. JONES
}

\begin{abstract}
Let $x, e \geqslant 0, u_{0}<\cdots<u_{d+e}$, and $h>0$ be real numbers. Let $f$ be a real valued function and let $\Delta(h ; u, w) f(x) h^{-d}$ be a difference quotient associated with a generalized Riemann derivative. Set $I=\left(x+u_{0} h, x+u_{d+e} h\right)$ and let $f$ have its ordinary $(d-1)$ st derivative continuous on the closure of $I$ and its $d$ th ordinary derivative $f^{(d)}$ existent on $I$. A necessary and sufficient condition that a difference quotient satisfy a mean value theorem (i.e., that there be a $\xi \in I$ such that the difference quotient is equal to $f^{(d)}(\xi)$ ) is given for $d=1$ and $d=2$. The condition is sufficient for all $d$. It is used to show that many generalized Riemann derivatives that are "good" for numerical analysis do not satisfy this mean value theorem.
\end{abstract}

1. Results. Let $f$ be a real valued function of a real variable. The $d$ th Riemann derivative of $f$ is

$$
R_{d} f(x):=\lim _{h \rightarrow 0} \frac{\sum_{i=0}^{d}\left(\begin{array}{l}
d \\
i
\end{array}\right)(-1)^{d-i} f(x+(-d / 2+i) h)}{h^{d}}
$$

The first two special cases

$$
R_{1} f(x)=\lim _{h \rightarrow 0} \frac{-f(x-h / 2)+f(x+h / 2)}{h}
$$

and

$$
R_{2} f(x)=\lim _{h \rightarrow 0} \frac{f(x-h)-2 f(x)+f(x+h)}{h^{2}}
$$

are the well-known symmetric and Schwarz derivatives $[6,10,11]$.

The generalized Riemann derivative is

$$
D_{d}(u, w) f(x):=\lim _{h \rightarrow 0} \frac{\Delta_{d}(h ; u, w) f(x)}{h^{d}},
$$

Received by the editors July 9, 1986.

1980 Mathematics Subject Classification (1985 Revision). Primary 26A24; Secondary 41A80, 65D25.

Key words and phrases. Generalized Riemann derivative, mean value theorem.

The research presented here was supported in part by grants to both authors from the Faculty Research and Development Fund of the College of Liberal Arts and Sciences, DePaul University.

$$
\text { 1987 American Mathematical Society }
$$
$0002-9939 / 87 \$ 1.00+\$ .25$ per page 
where

$$
\Delta_{d}(h ; u, w) f(x):=\sum_{i=0}^{d+e} w_{i} f\left(x+u_{i} h\right)
$$

here $e$ is a nonnegative integer which we will call the excess and the $w_{i}$ 's and $u_{i}$ 's are real numbers. Here we insist upon the $d+1$ consistency conditions

$$
\sum w_{i} u_{i}^{j}= \begin{cases}0, & j=0,1, \ldots, d-1, \\ d !, & j=d .\end{cases}
$$

$[1,2,7]$. For notational convenience we will always assume $u_{0}<u_{1}<\cdots<u_{d+e}$.

The most natural type of mean value theorem would say something like this. Let $I=\left[x+u_{0} h, x+u_{d+e} h\right]$ where $x$ and $h$ are fixed. If $D_{d} f(t)$ exists for every $t \in I$, then there is a $\xi$ interior to $I$ with

$$
\frac{\Delta_{d}(h ; u, w) f(x)}{h^{d}}=D_{d} f(\xi) .
$$

But this is not even true for $R_{1}$ as the choices $x=-1, h=3$, and $f(t)=|t|$ show [3].

It is possible that the only generalized Riemann derivative for which this mean value theorem holds is $d / d x$ itself. A more fruitful set of mean value theorems are those of the following type.

Statement $M(u, w)$. Fix $x$ and $h$ and set $I=\left[x+u_{0} h, x+u_{d+e} h\right]$. If $f^{(d-1)}(t)$ is continuous on $I$ and differentiable for all $t$ interior to $I$, then there is a $\xi$ interior to $I$ with $\Delta_{d}(h ; u, w) f(x) / h^{d}=f^{(d)}(\xi)$.

It is easy to see that any set of $d+1$ distinct points $\mu=\left\{\mu_{0}, \ldots, \mu_{d}\right\}$ has a unique $d$ th derivative associated to it via the correspondence $\mu \mapsto D_{\mu}$ where

$$
D_{\mu} f(x):=\lim _{h \rightarrow 0} \sum_{i=0}^{d} d ! \frac{\left[\Pi_{j \neq i}\left(\mu_{i}-\mu_{j}\right)\right]^{-1} f\left(x+\mu_{i} h\right)}{h^{d}}
$$

(see p. 182 of [1] or p. 12 of [3] for this). In particular, if $d=1$ then the unique two point derivative at $\left\{\mu_{0}, \mu_{1}\right\}$ is

$$
D_{\mu} f(x)=\lim _{h \rightarrow 0} \frac{f\left(x+\mu_{1} h\right)-f\left(x+\mu_{0} h\right)}{\left(\mu_{1}-\mu_{0}\right) h}
$$

and if $d=2$ then the unique three point derivative at $\left\{\mu_{0}, \mu_{1}, \mu_{2}\right\}$ is

$$
\begin{aligned}
& D_{\mu} f(x)=\lim _{h \rightarrow 0} \frac{1}{h^{2}}\left[\frac{2 f(x}{}+\mu_{0} h\right) \\
&\left.+\frac{2 f\left(x+\mu_{1} h\right)}{\left(\mu_{0}-\mu_{1}\right)\left(\mu_{0}-\mu_{2}\right)}+\frac{2 f\left(x+\mu_{2} h\right)}{\left(\mu_{2}-\mu_{0}\right)\left(\mu_{2}-\mu_{2}\right)}\right] .
\end{aligned}
$$

Lemma 1. Let $u=\left(u_{0}, \ldots, u_{d+e}\right)$ and $w=\left(w_{0}, \ldots, w_{d+e}\right)$ be given and let $D_{j}$ denote the unique generalized Riemann derivative associated with $\left(u_{j}, \ldots, u_{j+d}\right)$, $j=0, \ldots, e$. Then the $D$ associated with $u$ and $w$ is itself a generalized Riemann derivative if and only if there are $p_{j}, j=0, \ldots, e$, such that $\sum p_{j}=1$ and $D=\sum p_{j} D_{j}$.

We will prove this in $\$ 2$. 
THEOREM. Let $D=D_{d}(u, w)$ be a dth generalized Riemann derivative.

(i) If the $p_{i}$ associated to $D$ are all positive (so that $D$ is a convex combination of the $D_{j}$ 's $)$, then the mean value theorem $M(u, w)$ holds.

(ii) Conversely if $d=1$ or $d=2$ or $e=1$, and if any $p_{i}$ is negative, then Statement $M(u, w)$ is false.

The proof of (i) is easy. First if $e=0$ then $p_{0}=1$ and indeed the Theorem is a well-established numerical analysis fact [8]. If $e>0$ using this fact $e+1$ times we have numbers $\xi_{i}$ so that

$$
s=\frac{\Delta_{d}(h ; u, w) f(x)}{h^{d}}=\sum_{i=0}^{e} p_{i} f^{(d)}\left(\xi_{i}\right) .
$$

The right side is a convex combination of the numbers $\left\{f^{(d)}\left(\xi_{0}\right), \ldots, f^{(d)}\left(\xi_{e}\right)\right\}$ and hence $s$ lies between the smallest and the largest. But $f^{(d)}=\left(f^{(d-1)}\right)^{\prime}$ is an ordinary first derivative, hence is Darboux and therefore assumes the value $s$.

The proof of (ii) is longer so we will break it into three lemmas. These are contained in $\$ 2$.

APPliCATIONS. 1. Using Taylor expansion techniques, it is easy to see that

$$
\frac{\Delta_{d}(h ; u, w) f(0)}{h^{d}}=\sum_{j=0}^{p} c_{j} \frac{f^{(j)}(0)}{j !} h^{j-d}+O\left(h^{p+1-d}\right),
$$

where $p>d$ and $c_{j}=\sum_{i=1}^{d+e} w_{i} u_{i}^{j}, j=0,1, \ldots, p$. To yield a generalized Riemann derivative, the $c_{j}, j=0, \ldots, d$, must satisfy the consistency conditions (3). In [5] a difference quotient based on $d+e$ points was defined to be optimal if in addition to the consistency conditions (3) we also have $c_{j}=0$ for $j=d+1, \ldots, r$, with $r$ as large as possible, $\left|c_{r+1}\right|$ as small as possible, and $\left|u_{i}-u_{j}\right| \geqslant 1$ for $i \neq j$. (The last condition is for normalization [5].)

With this definition of optimal, the above theorem implies that no optimal first or second derivative with positive excess has the mean value property. This will be proved at the end of $\S 2$.

2. From the Theorem it follows that the first derivative

$$
\begin{aligned}
\lim _{h \rightarrow 0} & \frac{-27 f(x-2 h)+32 f(x+3 h)-5 f(x+6 h)}{120 h} \\
& =\frac{9}{8}\left(\lim _{h \rightarrow 0} \frac{f(x+3 h)-f(x-2 h)}{5 h}\right)-\frac{1}{8}\left(\lim _{h \rightarrow 0} \frac{f(x+6 h)-f(x+3 h)}{3 h}\right)
\end{aligned}
$$

does not have the mean value property. This derivative is optimal in some senses for numerical approximation (see [4]).

3. The first derivative

$$
\lim _{h \rightarrow 0} \frac{7 f(x+3 h)-13 f(x+4 h)+6 f(x+16 h / 3)}{h},
$$

whose interesting properties were investigated by Patrick O'Conner, is an example of a derivative not having the mean value property (see [3, pp. 26, 17-18]; this derivative was discovered in [9]). 
Question. Does the mean value theorem hold if $d \geqslant 3, e \geqslant 2$, and some $p_{i}$ is negative? In particular, does the mean value theorem hold for the excess 2 third derivative $D:=\frac{5}{8} D_{0}-\frac{1}{4} D_{1}+\frac{5}{8} D_{2}$, where for $i=0,1,2$,

$D_{i}:=\lim _{h \rightarrow 0} \frac{f(x+i h)+3 f(x+[i+1] h)-3 f(x+[i+2] h)+f(x+[i+3] h)}{h^{3}}$ ?

\section{Proofs.}

Proof of Lemma 1. Let $p_{0}, \ldots, p_{e}$ be real numbers with $\sum p_{i}=1$. Let $u_{0}<u_{1}<$ $\cdots<u_{d+e}$ be $d+1+e$ real numbers. For $j=0, \ldots, e$, let $D_{j}$ denote the unique generalized Riemann derivative based on $\left\{u_{j}, \ldots, u_{d+j}\right\}$, and let $D=\sum_{i=0}^{e} p_{i} D_{i}$. Then a quick check of the consistency condition shows that $D$ is also a $d$ th derivative.

Conversely, if

$$
D \sim \Delta=\sum_{j=0}^{d+e} w_{j} f\left(x+u_{j} h\right)
$$

and

$$
D_{j} \sim \Delta_{j}=\sum_{k=j}^{j+d} w_{k j} f\left(x+u_{k} h\right) \text { for } j=0,1, \ldots, e
$$

(recall $w_{k j}=d !\left[\prod_{l=j, l \neq k}^{j+d}\left(u_{k}-u_{l}\right)\right]^{-1}$ ), then $w_{j}=\sum_{i=0}^{j} p_{i} w_{j-i, i}, j=0, \ldots, e$. This allows the $p_{i}$ 's to be found recursively as

$$
p_{0}:=w_{0} / w_{00}, \quad p_{j}:=\left(w_{j}-\sum_{i=0}^{j-1} p_{i} w_{j-1, i}\right) / w_{j, j}, \quad j=1,2, \ldots, e .
$$

Now let $Z=\Delta-\sum p_{j} \Delta_{j}$. We must show $Z=0$. From the definition of the $p_{j}$ 's, $Z=\sum_{j=e+1}^{e+d} c_{j} f\left(x+u_{j} h\right)$ for some numbers $c_{j}$. Testing $Z$ on the functions 1 , $t, \ldots, t^{d-1}$ at the point $x=0$ and noting that $D$ and all of the $D_{j}$ satisfy the first $d$ conditions in (3), we get $\sum c_{j} u_{i}^{j}=0, j=0, \ldots, d-1$. Since the Vandermonde matrix $\left(u_{i}^{j}\right)$ is invertible, $Z=0$. Applying $Z$ to the function $t^{d}$ at $x=0$, noting that $D$ and all $D_{j}$ satisfy the last equation in (3), and that $Z=0$, we get that $\sum p_{i}=1$. Observe that the equations defining the $p_{j}$ 's are quite explicit and simple.

Lemma 2. Let $D=D_{1}(u, w) f(x)$ be a generalized first derivative, so that $D$ has a unique representation as a linear combination of $e+1$ two point first derivatives: $D=\sum_{j=0}^{e} p_{j} D_{j}$. If for some $j, p_{j}<0$, then the mean value theorem fails.

Proof. The proof will consist of constructing a function for which the mean value theorem fails. Define $f$ inductively. Let $f\left(u_{0}\right)=0$. Having defined $f\left(u_{i-1}\right)$, set $f\left(u_{i}\right)=f\left(u_{i-1}\right)$ if $p_{i-1}$ is $\leqslant 0$, and $f\left(u_{i}\right)=f\left(u_{i-1}\right)+\left(u_{i}-u_{i-1}\right)$ if $p_{i-1}>0$. Note $f$ is not linear on $\left[u_{0}, u_{e+1}\right]$ since at least one $p_{j}$ is negative and $\sum p_{i}=1$.

We now extend the domain of $f$ from $\left\{u_{0}, \ldots, u_{e+1}\right\}$ to all of $\mathbf{R}$ by making $f$ linear on each interval $\left[u_{i}, u_{i+1}\right], i=1, \ldots, e-1$, linear on $\left(-\infty, u_{1}\right]$, linear on $\left[u_{e}, \infty\right)$, and continuous on $\mathbf{R}$. Since

$$
\Delta_{j}(h ; u) f(x)=\frac{f\left(x+u_{j+1} h\right)-f\left(x+u_{j} h\right)}{\left(u_{j+1}-u_{j}\right) h},
$$


it follows that whenever $p_{j} \leqslant 0$ we have $\Delta_{j}(1 ; u) f(0)=0$ and whenever $p_{j}>0$ we have $\Delta_{j}(1 ; u) f(0)=1$. Consequently $\Delta(1 ; u) f(0)=\sum p_{j} \Delta_{j} f(0)>1$. Finally, round the corners of $f$ to produce a differentiable function $g$ with $g^{\prime}(x) \leqslant 1$ for all $x$, but $\Delta(g)>1$, so that the mean value theorem fails for $g$.

Lemma 3. Let $D=D_{2}(u, w) f(x)$ be a generalized second derivative, so that $D$ has a unique representation as a linear combination of $e+1$ three point second derivatives, $D=\sum_{j=0}^{e} p_{j} D_{j}$. If for some $j, p_{j}<0$, then the mean value theorem fails.

Proof. Let $D$ be given with some $p_{j}<0$. Fix $x=0$ and $h=1$. Let $\Delta_{j}:=\sum_{i=j}^{j+2} \omega_{i j} f\left(u_{i}\right)$ and $\Delta:=\sum w_{i} f\left(u_{i}\right)$ so that $\Delta=\sum p_{j} \Delta_{j}$. Let $\mathscr{P}=\left\{j: p_{j}>0\right\}$ and $\mathscr{N}=\left\{j: p_{j}<0\right\}$. Then $\sum_{j \in \mathscr{P}} p_{j}+\sum_{j \in \mathscr{N}} p_{j}=1$, so $\sum_{j \in \mathscr{N}} p_{j}<0$ implies $\sum_{j \in \mathscr{P}} p_{j}>$ 1.

We prove Lemma 3 by constructing a function $f$ satisfying $\Delta>1$ and $f^{\prime \prime}(x) \leqslant 1$ for all $x \in\left[u_{0}, u_{e+2}\right]$. The function $f$ will be created by rounding the corners of a continuous, piecewise twice differentiable function $g$.

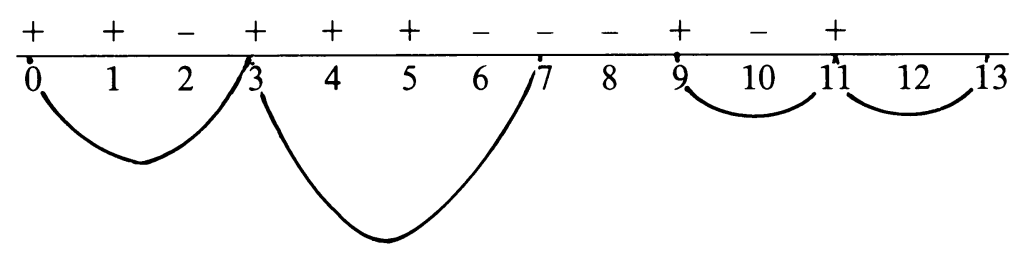

FIGURE 1

To understand how $g$ is constructed in general first consider an example (Figure 1). In Figure 1 the point labeled 4 is $u_{4}$, and the + sign above the 4 indicates that $p_{4}$ is positive, etc. The function $g$ is defined by

$$
g(x)=\left(\begin{array}{ll}
.5\left(x-u_{0}\right)\left(x-u_{3}\right), & u_{0} \leqslant x \leqslant u_{3}, \\
.5\left(x-u_{3}\right)\left(x-u_{7}\right), & u_{3} \leqslant x \leqslant u_{7}, \\
0, & u_{7} \leqslant x \leqslant u_{9}, \\
.5\left(x-u_{9}\right)\left(x-u_{11}\right), & u_{9} \leqslant x \leqslant u_{11}, \\
.5\left(x-u_{11}\right)\left(x-u_{13}\right), & u_{11} \leqslant x \leqslant u_{13} .
\end{array}\right.
$$

Observe that $g^{\prime \prime}(x) \in\{0,1\}$ when $x \notin\left\{u_{3}, u_{7}, u_{9}, u_{11}\right\}$.

We have $\Delta_{0}=\Delta_{1}=\Delta_{3}=\Delta_{4}=\Delta_{5}=\Delta_{9}=\Delta_{11}=1$. To see this note for example that from the viewpoint of $\Delta_{4}=\omega_{44} g\left(u_{4}\right)+\omega_{54} g\left(u_{5}\right)+\omega_{64} g\left(u_{6}\right)$ the function $g$ "is" the quadratic polynomial $.5\left(x-u_{3}\right)\left(x-u_{7}\right)$ and $\Delta_{4}\left(.5 x^{2}+\cdots\right)=$ $\left(.5 x^{2}+\cdots\right)^{\prime \prime}=1$. Similarly $\Delta_{7}=0$. Next observe that the line joining $\left(u_{2}, g\left(u_{2}\right)\right)$ to $\left(u_{4}, g\left(u_{4}\right)\right)$ passes below the point $\left(u_{3}, g\left(u_{3}\right)\right)=\left(u_{3}, 0\right)$ so the quadratic $q$ interpolating these three points has negative second derivative. Since $g=q$ from the viewpoint of $\Delta_{2}=\Delta_{2}\left(u_{2}, u_{3}, u_{4}\right)$, it follows that $\Delta_{2}<0$. Similarly, $\Delta_{6}, \Delta_{8}$, and $\Delta_{10}$ are also negative. Totalling up,

$$
\Delta=\sum_{i \in\{0,1,3,4,5,9,11\}} p_{i} \Delta_{i}+\sum_{j \in\{2,6,7,8,10\}} p_{j} \Delta_{j}>\sum_{i \in\{0,1,3,4,5,9,11\}} p_{i}
$$




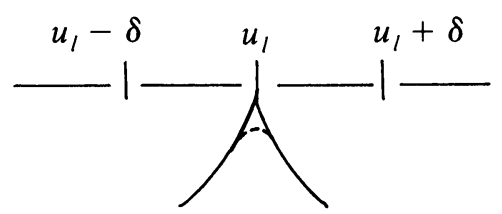

Figure 2

since in the first sum each $\Delta_{i}=1$, while in the second sum each $p_{j}<0$ and each $\Delta_{j} \leqslant 0$. Hence $\Delta>\sum_{i \in \mathscr{P}} p_{i}>1$.

We now show how to construct such a function $g$ whenever $\mathscr{N}$ is not empty. We suggest that the reader test the construction on the above example to see how it works.

Let $j_{1}$ be the smallest index in $\mathscr{P}$. Let $g(x)=0$ for $x \in\left[u_{0}, u_{j_{1}}\right]$. If $\mathscr{N}$ has any numbers larger than $j_{1}$, let $k_{1}=\min \left\{n \in \mathscr{N} \mid n>j_{1}\right\}+1$; otherwise let $k_{1}=e+2$. Let $g(x)=.5\left(x-u_{j_{1}}\right)\left(x-u_{k_{1}}\right)$ for $x \in\left[u_{j_{1}}, u_{k_{1}}\right]$. If $\mathscr{P}$ has any members $\geqslant k_{1}$, let $j_{2}$ be the least one; otherwise let $j_{2}=e+2$. Let $g(x)=0$ for $x \in\left[u_{k_{1}}, u_{j_{2}}\right]$. If $\mathscr{N}$ has any members larger than $j_{2}$, let $k_{2}=\min \left\{n \in \mathscr{N} \mid n>j_{2}\right\}+1$; otherwise let $k_{2}=e+2$. Let $g(x)=.5\left(x-u_{j_{2}}\right)\left(x-u_{k_{2}}\right)$ on $\left[u_{j_{2}}, u_{k_{2}}\right]$. Proceed inductively. The process terminates when some $j_{i}$ or $k_{i}=e+2$. The process does terminate since $j_{i}<k_{i} \leqslant j_{i+1}$.

Having constructed $g$, we now show that $\Delta(g)>1$. It suffices to show that $\Delta_{i} \leqslant 0$ if $p_{i} \in \mathscr{N}$ and $\Delta_{i}=1$ if $p_{i} \in \mathscr{P}$, for then

$$
\Delta=\sum_{i \in \mathscr{P}} p_{i} \Delta_{i}+\sum_{i \in \mathscr{N}} p_{i} \Delta_{i} \geqslant \sum_{i \in \mathscr{P}} p_{i} \Delta_{i}=\sum_{i \in \mathscr{P}} p_{i}>1
$$

Let $l \in \mathscr{N}$. Then either $l \leqslant j_{1}-1, l \in\left[k_{\nu}-1, j_{\nu+1}\right)$ for some $\nu$, or $l>j_{p}$ where $j_{p}$ is the largest member of $\mathscr{P}$. If $l \leqslant j_{1}-2$ or $l \in\left[k_{\nu}-1, j_{\nu+1}-1\right)$ or $l \in\left[j_{p}+2, e\right]$, then $g\left(u_{e}\right)=g\left(u_{e+1}^{\dot{*}}\right)=g\left(u_{e+2}\right)=0$ and $\Delta_{l}=0$. If $l=j_{1}-1$ or $j_{v+1}-1$, then $g\left(u_{e}\right) \leqslant 0, g\left(u_{e+1}\right)=0$, and $g\left(u_{e+2}\right)<0$ so that the quadratic $q$ passing through the three points $\left(u_{k}, g\left(u_{k}\right)\right), k=e, e+1, e+2$, is concave, whence $\Delta_{e}=q^{\prime \prime}<0$. Finally if $l=j_{p}+1, g\left(u_{e}\right)<0, g\left(u_{e+1}\right)=g\left(u_{e+2}\right)=0$ so that $\Delta_{l}<0$.

If now $l \in \mathscr{P}$, then $g$ agrees with a quadratic of the form $q(x)=.5(x-\alpha)(x-\beta)$ at $x=u_{l}, u_{e+1}$, and $u_{e+2}$ so that $\Delta_{l}=q^{\prime \prime}=1$.

Let $\varepsilon_{0}=\Delta-1$. We now construct $f$ as a twice differentiable perturbation of $g$. Any point of $\left(u_{0}, u_{e+2}\right)$ where $g^{\prime \prime}$ does not exist is of the form $u_{l}$ where either (i) $l \in\left\{j_{\nu}\right\} \cap\left\{k_{\nu}\right\}\left(u_{3}\right.$ and $u_{11}$ in the example), (ii) $l \in\left\{k_{\nu}\right\} \backslash\left\{j_{\nu}\right\}\left(u_{7}\right.$ in the example), or (iii) $l \in\left\{j_{\nu}\right\} \backslash\left\{k_{\nu}\right\}$ ( $u_{9}$ in the example).

In case (i), $g$ looks like the solid curve in Figure 2.

Let $f$ be a rounded version of $g$ on $\left[u_{l}-\delta, u_{l}+\delta\right]$ (the dotted curve in Figure 2). Then on $\left[u_{l}-\delta, u_{l}+\delta\right] \cap\{x: f(x) \neq g(x)\}$ the slope of $f$ decreases steadily so $f^{\prime \prime}<0$. Pick $\delta$ so small that

$$
-f\left(u_{l}\right)<\frac{\varepsilon_{0}}{2 e\left|w_{l}\right|}
$$



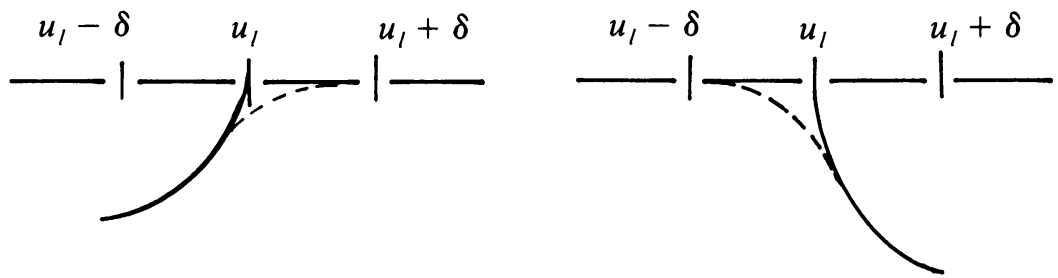

FIGURE 3

and also so small that

$$
\delta<\frac{1}{2} \min \left\{u_{l}-u_{l-1}, u_{l+1}-u_{l}\right\} .
$$

Cases (ii) and (iii) are similar. The pictures are given in Figure 3.

Again we get $f^{\prime \prime}<0$ on $\left[u_{l}-\delta, u_{l}+\delta\right] \cap\{x: f(x) \neq g(x)\}$ in both cases. Again we choose $\delta$ small enough to force (1) and (2). Define $f(x)=g(x)$ on the remainder of $\left[u_{0}, u_{e+2}\right]$. Then $f^{\prime \prime}(x) \leqslant 1$ everywhere on $\left(u_{0}, u_{e+2}\right)$ and $f^{\prime}$ is continuous on $\left[u_{0}, u_{e+2}\right]$ (with the usual convention that $f^{\prime}\left(u_{0}\right):=f^{\prime+}\left(u_{0}\right), f^{\prime}\left(u_{e+2}\right):=f^{\prime-}\left(u_{e+2}\right)$ ). However $\Delta(f)=\Delta(g)-\sum w_{l} f\left(u_{l}\right)>\left(1+\varepsilon_{0}\right)-\Sigma\left(\varepsilon_{0} / 2 e\right)$ where the sum is over the discontinuities of $g^{\prime \prime}$. Since there are at most $e$ such $u_{l}$ 's, it follows that $\Delta(f)>1+\varepsilon_{0} / 2>1$ and hence $\Delta(f) \notin \operatorname{Range}\left(f^{\prime \prime}\right) \subseteq(-\infty, 1]$. Therefore the mean value theorem fails for this $f$.

Lemma 4. Let $D=D_{d}(u, w) f(x)$ be a generalized dth derivative with excess 1, where $u_{0}<\cdots<u_{d+1}$, so that $D$ has a unique representation as a linear combination of two $d+1$ point dth derivatives, $D=p_{0} D_{0}+p_{1} D_{1}$. If one $p_{j}$ is negative, then the mean value theorem is false for $D$.

Proof. Let $D_{0}$ be the unique $d$ th derivative based on $\left\{u_{0}, \ldots, u_{d}\right\}$ and $D_{1}$ the one based on $\left\{u_{1}, \ldots, u_{d+1}\right\}$. Let $D=p_{0} D_{0}+p_{1} D_{1}$. Fix $x_{0}=0, h=1$, and let $\Delta$, $\Delta_{0}$, and $\Delta_{1}$ be the respective corresponding differences. First suppose $p_{0}<0$. Let $f=\left(x-u_{1}\right)^{d} / d$ ! on $\left[u_{1}, u_{d+1}\right]$, and $f(x)=0$ or $\left[u_{0}, u_{1}\right]$. Now

$$
\Delta_{0}\left(\frac{1}{d !}\left(x-u_{1}\right)^{d}\right)=\frac{d !}{\prod_{i=1}^{d}\left(u_{0}-u_{i}\right)} \cdot \frac{1}{d !}\left(u_{0}-u_{1}\right)^{d}+\cdots
$$

has positive first term since $\operatorname{sgn}\left(\prod_{i=1}^{d}\left(u_{0}-u_{i}\right)\right)=\operatorname{sgn}\left(\left(u_{0}-u_{1}\right)^{d}\right)$, while

$$
\Delta_{0}(f)=\frac{d !}{\prod_{i=1}^{d}\left(u_{0}-u_{i}\right)} f\left(u_{0}\right)+\cdots
$$

has 0 first term. Since all other terms are equal, $\Delta_{0}(f)<\Delta_{0}\left(\left(x-u_{1}\right)^{d} / d\right.$ !) $=1$. Write $\Delta_{0}(f)=1-\eta$ where $\eta>0$. Then $\Delta(f)=p_{0}(1+\eta)+p_{1}(1)=p_{0}+p_{1}-$ $p_{0} \eta=1-p_{0} \eta>1$ since $p_{0}<0$ and $\eta>0$. However, for $x \neq u_{1}$,

$$
f^{(d)}(x)=H\left(x-u_{1}\right)= \begin{cases}0, & x<u_{1} \\ 1, & x>u_{1}\end{cases}
$$


We must smooth $f$. Let

$$
H_{\varepsilon}(x)=\left(\begin{array}{ll}
0, & x<0 \\
x / \varepsilon, & 0 \leqslant x \leqslant \varepsilon \\
1, & x>\varepsilon
\end{array}\right.
$$

Then let

$$
f_{\varepsilon}(x)=\int_{0}^{x-u_{1}} \frac{\left(x-u_{1}-t\right)^{d-1}}{(d-1) !} H_{\varepsilon}(t) d t
$$

If $x \in\left[u_{0}, u_{d+1}\right]$

$$
\left|f(x)-f_{\varepsilon}(x)\right| \leqslant \int_{0}^{\varepsilon} \frac{\left(x-u_{1}-t\right)^{d-1}}{(d-1) !} d t=O(\varepsilon),
$$

so that if $\varepsilon$ is small enough, $\Delta\left(f_{\varepsilon}\right)>1$. However, $f_{\varepsilon}^{(d)}(x)=H_{\varepsilon}\left(x-u_{1}\right)$ has range contained in $[0,1]$, so the mean value theorem fails for $p_{0}$ negative.

If $p_{0}$ is nonnegative, then $p_{1}<0$. In this case let $f(x)=\left(x-u_{d}\right)^{d} / d$ ! on $\left[u_{0}, u_{d}\right]$ and $f(x)=0$ on $\left[u_{d}, u_{d+1}\right]$. As above, $\Delta(f)>1, f^{(d)}(x) \in\{0,1\}$ for $x \neq u_{\alpha}$, and a smoothed version of $f$ then becomes the required counterexample.

Proof FOR APPLICATION 1. Let $\Delta(h ; u, w) f(x)=\sum_{i=1}^{n} w_{i} f\left(x+u_{i} h\right)$ be the difference associated to an optimal first derivative based on $n \geqslant 3$ points. From [5] we have that if $n=2 k, k=2,3, \ldots$, then the optimal choice of $u$ is $u=\left(-\left(k-\frac{1}{2}\right)\right.$, $\left.-\left(k-\frac{3}{2}\right), \ldots,-\frac{1}{2}, \frac{1}{2}, \ldots, k-\frac{1}{2}\right)$; while if $n=2 k+1, k=1,2,3, \ldots$, then the optimal choice of $u$ is $u=\left(\alpha_{n}-k, \alpha_{n}-k+1, \ldots, \alpha_{n}+k\right)$ where $\frac{1}{2}<\alpha_{n}<\frac{1}{2}+$ $1 / 4 n, n=3,5,7, \ldots$. Fix $n$. Let

$$
R_{1}(x):=\prod_{i=2}^{n}\left(x-u_{i}\right) \text { and } R_{2}(x):=\left(x-u_{1}\right) \prod_{i=3}^{n}\left(x-u_{i}\right) .
$$

On the one hand, logarithmic differentiation of $R_{j}$ and the fact that the optimal choice of $u$ satisfies identity $\sum u_{i}^{-1}=0$ (see [5, p. 157]) yield $R_{j}^{\prime}(0)=u_{j}^{-1} \Pi_{i \neq j}\left(-u_{i}\right)$, $j=1,2$; while on the other hand from $\Delta(1 ; u, w) R_{j}(0)=R_{j}^{\prime}(0), j=1,2$ (see [5]), we have $R_{j}^{\prime}(0)=w_{j} R_{j}\left(u_{j}\right), j=1,2$. This yields formulas for $w_{1}$ and $w_{2}$ in terms of $k$. From these and the proof of Lemma 1 we get

$$
\frac{p_{2}}{p_{1}}=1+\frac{w_{2}}{w_{1}}=1-\left(\frac{u}{u+1}\right)(n-1)
$$

where $u=-k+\frac{1}{2}$ when $k$ is even and $\alpha_{n}-k$ when $k$ is odd. This is easily shown to be negative. Since one of $\left\{p_{1}, p_{2}\right\}$ must be negative, by the Theorem of $\S 1$ each optimal $n$ point first derivative, $n \geqslant 3$, fails to satisfy a mean value theorem.

For the second derivative case let $\Delta=\sum_{i=1}^{n} w_{i} f\left(x+u_{i} h\right)$ be the difference associated to an optimal second derivative based on $n \geqslant 4$ points. Again using the results from [5] we have if $n=2 k+1, k=2,3, \ldots$, then the optimal choice of $u$ is $u=(-k,-k+1, \ldots, k-1, k)$; while if $n=2 k, k=2,3,4, \ldots$, then the optimal choice of $u$ is $u=\left(\beta_{n}-k, \beta_{n}-k+1, \ldots, \beta_{n}+k-1\right)$ where $\beta_{4}=1.145, \beta_{6}=$ 1.085 , and $1<\beta_{n}<1+1 / 2 n, n=8,10, \ldots$. 
Computing $\left(\log R_{j}\right)^{\prime \prime}$ and using the fact that the optimal choice of $u$ satisfies the identity $\sum_{i \neq j}\left(u_{i} u_{j}\right)^{-1}=0$ (see [5, pp. 162-163]), we obtain

$$
R_{j}^{\prime \prime}(0)=\frac{2 \prod_{i=1}^{n}\left(-u_{i}\right)\left(u_{j} \sum_{i=1}^{n} u_{i}^{-1}-1\right)}{u_{j}^{3} \prod_{i \neq j}\left(u_{j}-u_{i}\right)}, \quad j=1,2 .
$$

On the other hand from $\Delta(1 ; u, w) R_{j}(0)=R_{j}^{\prime \prime}(0)$ we have $R_{j}^{\prime \prime}(0)=w_{j} R_{j}\left(u_{j}\right), j=$ 1,2 . This yields formulas for $w_{1}$ and $w_{2}$ in terms of $u$. From these and the proof of Lemma 1 we get

$$
\frac{p_{2}}{p_{1}}=2+\frac{w_{2}}{w_{1}}=2-(n-1)\left(\frac{u}{u+1}\right)^{3}\left(\frac{1-(u+1) \sum_{i=1}^{n} u_{i}^{-1}}{1-u \sum_{i=1}^{n} u_{i}^{-1}}\right),
$$

where $u=-k$ if $n$ is odd and $u=\beta_{n}-k$ if $k$ is even. It can now be shown that $p_{2} / p_{1}<0$. (To cio this, we needed three cases: $n$ odd, $n$ even and at least 6 , and $n=4$.) Again finish by applying the Theorem of $\$ 1$.

\section{REFERENCES}

1. J. M. Ash, Generalizations of the Riemann derivative, Trans. Amer. Math. Soc. 126 (1967), 181-199.

2. __ A characterization of the Peano derivative, Trans. Amer. Math. Soc. 149 (1970), 489-501.

3. W Very generalized Riemann derivatives, generalized Riemann derivatives and associated summability methods, Real Anal. Exchange 11 (1985-86), 10-29.

4. J. M. Ash and R. L. Jones, Optimal numerical differentiation using three function evaluations, Math. Comp. 37 (1981), 159-167.

5. J. M. Ash, S. Janson, and R. L. Jones, Optimal numerical differentiation using $n$ function evaluations, Estrato da Calcolo 21 (1984), 151-169.

6. G. Cantor, Gesammelte Abhandlungen, Georg Olms, Hildesheim, 1962, 80-83. Beweis, das eine für jeden reellen Wert von $x$ durch eine trigonometrische Reihe gegebene Funktion $f(x)$ sich nur auf eine einzige Weise in dieser Form darstellen lässt, Crelles J. Math. 72 (1870), 139-142.

7. A. Denjoy, Sur l'intégration des ccefficients différentiels d'ordre supérieur, Fund. Math. 25 (1935), $273-326$.

8. E. Isaacson and H. B. Keller, Analysis of numerical methods, Wiley, New York; 1966.

9. P. J. O'Connor, Generalized differentiation of functions of a real variable, Ph.D. dissertation, Wesleyan Univ., Middletown, Conn., 1969.

10. B. Riemann, Über die Darstellbarkeit einer Function durch eine trigonometrische Reihe, Ges. Werke, 2. Auf1., Leipzig, 1892, pp. 227-271; also Dover, New York, 1953.

11. A. Zygmund, Trigonometric series, Vols. I and II, Cambridge Univ. Press, Cambridge, 1959.

Mathematical Sciences Department, DePaul University, Chicago, Illinois 60614 